\title{
La competencia cultural, una clave para mejores resultados en salud
}

\author{
The cultural competence, a key for better health outcomes \\ A competência cultural, é peça chave para obter melhores \\ resultados em saúde
}

La antropología de la salud, la enfermería transcultural entre otras áreas de conocimiento, han mostrado evidencia de cómo los aspectos culturales de las personas, familias o comunidades influyen en la forma de interpretar, explicar, reconocer y cuidar la salud, la enfermedad, la discapacidad, el dolor y la muerte. Este hecho impacta de forma considerable en cómo las personas buscan y responden a los servicios de salud y cómo se podría desarrollar la práctica en salud. La Competencia Cultural en Salud (CCS) sugiere la habilidad, el conocimiento, la sensibilidad de los proveedores y organizaciones de salud para comprender y responder de forma eficaz, efectiva, segura, coherente y sensible a la cultura de las personas, familias y comunidades.

Colombia es un país multicultural, pluriétnico y multilingüe reconocido en la Constitución Política de 1991. El fenómeno de desplazamiento interno con 6.9 millones de personas (1985-2015) reconfigura el mapa del país con un grupo social heterogéneo con dolorosos rasgos en común. Actualmente, un profesional de la salud en cualquier ámbito de la atención se encuentra con esa diversidad cultural y teóricamente debería estar en capacidad de responder a la misma. Sin embargo, estudios realizados por el grupo de investigación que lidero, reportan a usuarios del sistema de salud de comunidades rurales y urbanas con narrativas como: "en el hospital uno no vale nada ... lo mandan de aquí para allá, le hacen cosas y dicen cosas que uno no entiende ... pero toca dejarse"; "allá le dicen todo es por su bien y mire quedé sin las piernas ... le echan a uno cuchillo y ¡listo! Uno verá que hace"; "yo creo en la gente del hospital, pero hablan que uno no entiende, le dan una comida muy malita y le sacan sangre a toda hora". De otro lado, proveedores de cuidado dicen: "no hay tiempo para hablar con el paciente, son muchos trámites"; "cuando son testigos de Jehová ya se sabe el problema con las transfusiones de sangre hay protocolos a seguir... en general uno hace su trabajo, les dice lo que está pasando y lo que deben hacer".

Desde el surgimiento de la CCS en los años 70 a la fecha se señala su potencial para mejorar la calidad de la atención y los resultados en salud. La evidencia apunta a la mejora en los resultados clínicos de los pacientes (1-4) aumento de la confianza del paciente en el profesional, satisfacción con la atención y adherencia a la dieta (3, 5-6,); mejora en conocimientos, actitudes y comportamientos sobre su enfermedad, hacia estilos de vida saludables, autoeficacia y prácticas de cuidado, mayor aceptación de ayuda, adherencia al tratamiento y menores conflictos en la toma de decisiones con respecto a éste(2-4).

La CCS viene llamando la atención de las autoridades de salud, profesionales y educadores de diversos países como una estrategia para mejorar la calidad y los resultados en salud, convirtiéndose en una característica clave en los estándares y procesos de acreditación para la atención en salud. En Europa hay iniciativas para el desarrollo de "Equity Standars" para hacer frente a las disparidades en salud; en Estados Unidos se han mejorado los "National Standards on Culturally and Linguistically Appropriate Services" desarrollados por la Oficina de Minorías en Salud y la "Joint Commission", quienes han desarrollado 
estándares para las organizaciones de salud dirigida a la comunicación eficaz, la competencia cultural y el cuidado centrado en el paciente(7).

En el mundo diversas organizaciones de Enfermería, Fisioterapia, Fonoaudiología, Medicina, Psicología, Trabajo Social, incorporan la CCS para proporcionar atención adecuada a los pacientes con diversos valores, creencias y comportamientos, incluyendo las necesidades sociales, culturales y lingüísticas (8). En América Latina es incipiente la incorporación de la CCS. México y Perú han puntualizado aspectos en la legislación en salud y en la práctica profesional(9-10). Colombia cuenta con un marco legal que reconoce la diversidad cultural desde la constitución política, la ley de talento humano, los códigos de ética médica y de enfermería; sin embargo, la obligación de proteger esa diferencia cultural en salud, con lineamientos como los internacionales aún está en mora. Algunos avances se vienen realizando en diferentes regiones(11). Desde nuestro grupo de investigación hemos: conocido las prácticas en salud en estudios cualitativos con poblaciones de personas ancianas vulnerables, mestizas y afrodescendientes en contextos urbanos y rurales de diversos municipios de Colombia (12-13); desarrollado y evaluado formas de atención culturalmente adaptadas (1416) e iniciamos a estudiar sobre la CCS de profesionales de la salud.

La CCS es prometedora para mejorar los conocimientos, actitudes y prácticas de atención de los profesionales (17), por lo que se requiere formación específica para una práctica efectiva y culturalmente competente (3). En 2000, el Comité de Enlace en Educación Médica presentó el siguiente estándar para la competencia cultural: "Docentes y estudiantes deben demostrar una comprensión de la manera en que la gente de diversas culturas y sistemas de creencias perciben la salud y la enfermedad y responden a diversos síntomas, enfermedades y tratamientos. Los estudiantes de medicina deben aprender a reconocer y a abordar de forma adecuada el género y los prejuicios culturales en la prestación de la atención de la salud, considerando en primer lugar la salud del paciente"(18).

En el ambiente internacional hay evidencia de la inclusión de la CCS en los currículos en la formación de profesionales de la salud y de entrenamientos para profesionales con estudios de alto nivel de evidencia (7, 19-22). En Europa el proyecto "Intercultural Education of Nurses and Health Professionals in Europe" (IENE) ha desarrollado 4 versiones promoviendo la CCS (23-24) y en el IENE 4 generó un modelo de desarrollo de modelos del rol para la competencia cultural y el cuidado culturalmente compasivo(22, 25).

América Latina y en especial Colombia necesitan emprender el desafío para responder a las diferencias no solo étnicas, sino a la diversidad religiosa, sexual, socioeconómica y regional que marca los modos de vida, incluso en las mismas regiones de un país. Este proceso pasa por el reconocimiento de la diversidad entre los proveedores de atención en salud con un mundo marcado por su herencia cultural y profesional. Además, delimitado culturalmente por el proyecto económico y político dominante en el sector salud. Hay evidencia de la necesidad de tomar la CCS como una forma de "garantizar que todas las personas reciban atención sanitaria equitativa, un cuidado eficaz, en particular aquellos de diversos orígenes culturales y lingüísticos... sigue siendo una estrategia importante para hacer frente a las desigualdades en salud" (7) y en la búsqueda de mejores resultados en salud. Este es un objetivo de la red de cuidado cultural de la salud, nacida en el 2006 y liderada desde nuestro grupo, como una forma de debatir cómo la CCS puede mejorar los resultados en salud (26).

\section{Lucero López-Díaz, Doctora en Enfermería., MSc., Enf Profesora Titular Universidad Nacional de Colombia - Facultad de Enfermería Líder del Grupo de Investigación en Cuidado Cultural de la Salud Reconocida como Transcultural Scholar (2016) por la Transcultural Nursing Society Correo electrónico: allopezdi@unal.edu.co}

\section{Referencias}

1. Thom DH, Tirado MD, Woon TL, McBride MR. Development and evaluation of a cultural competency training curriculum. BMC Med Educ. 2006; 6: 38.

2. Zeh P, Sandhu HK, Cannaby a. M, Sturt J a. The impact of culturally competent diabetes care interventions for improving diabetes-related outcomes in ethnic minority groups: A systematic review. Diabet Med. 2012; 29(10): 1237-52.

3. Truong M, Paradies Y, Priest N. Interventions to improve cultural competency in healthcare: a systematic review of reviews. BMC Health Serv Res. 2014; (14): 99.

4. Ruggiero L, Riley BB, Hernandez R, Quinn LT, Gerber BS, Castillo A, et al. Medical Assistant Coaching to Support Diabetes Self-Care Among Low-Income Racial/Ethnic Minority Populations: Randomized Controlled Trial. West J Nurs Res [Revista en internet]. 2014; 36(9): 1052-73. Disponible en: http://wjn .sagepub.com/cgi/doi/10. 1177/0193945914522862.

5. Melkus GDE, Spollett G, Jefferson V, Chyun D, Tuohy B, Robinson T, et al. A culturally competent intervention of education and care for black women with type 2 diabetes. Appl Nurs Res. 2004; 17(1): 10-20.

6. Tucker CM, Marsiske M, Rice KG, Nielson JJ, Herman K. Patient-centered culturally sensitive health care: Model Testing and Refinement. Health Psychol. 2011; 30(3): 342-50.

7. Horvat L, Horey D, Romios P, Kis-Rigo J. Cultural competence education for health professionals. Cochrane database Syst Rev. 2014;

8. Osorio-Merchán MB, López Díaz AL. Competencia cultural en salud: necesidad emergente en un mundo globalizado. Index de Enfermería [Revista en internet]. Fundación Index; 2008 ;17(4): 266-70. Disponible en: http://scielo.isciii.es/ scielo.php?script= 
sci_arttext\&pid=S1132-12962008004400110\&Ing= es\&nrm=iso\&tlng=es.

9. Salaverry O. Interculturalidad en Salud. Rev Peru Med Exp Salud Publica. 2010; 27(1): 80-93.

10. Zavaleta A. La competencia cultural y la prestación de servicios de salud a poblaciones latinas. Rev Int Ciencias Soc y Humanidades [Revista en internet]. 2006;XVI(2):163-81. Disponible en: http://www. redalyc.org/articulo.oa?id=65416207\nhttp:// communityforchildren.org/files/Zavaleta_La_Competen cia_Cultural_y_La_Prestacion_de_Servicios_de_Salud .pdffnhttp://www.redalyc.org/pdf/654/65416207.pdf.

11. Herrero-Hahn R, Rojas JG, Ospina-Diaz JM, MontoyaJuarez R, Restrepo-Medrano JC, Hueso-Montoro C. Cultural Adaptation and Validation of the Cultural SelfEfficacy Scale for Colombian Nursing Professionals. J Transcult Nurs [Revista en internet]. 2015; (November). Disponible en: http://tcn.sagepub.com/cgi/doi/10. $1177 / 1043659615613419$.

12. López-Díaz L, Castellanos-Soriano F, Muñoz-Torres E. Cuidado popular de familias con un adulto mayor sobreviviente del primer accidente cerebrovascular. Aquichan [Revista en internet]. 2016; 16(2): 169-78. Disponible en: http://aquichan.unisabana.edu.co/ index.php/aquichan/article/view/4594/pdf.

13. López L, Cataño N, López H, Velásquez V. Diversidad cultural de sanadores tradicionales afrocolombianos: preservacióan y conciliacióan de saberes. Rev Aquichan [Revista en internet]. 2011; 11(3): 287-304. Disponible en: http://search. ebscohost.com/login.aspx?direct= true \&db=a9h\&AN=74741198\&lang=es\&site=ehost-live.

14. Velásquez V, López L, López $H$, Cataño N, Muñoz E. Efecto de un programa educativo para cuidadores de personas ancianas: una perspectiva cultural. Rev Salud Pública [Revista en internet]. 2011; 13(4): 610-9. Disponible en: http://www.scielo.org.co/pdf/rsap/ v13n4/v13n4a06.pdf.

15. López L, Barreto Zorza YM, Maluche A. Dolor en personas ancianas afrodescendientes: resultados cualitativos de un programa domiciliario adaptado culturalmente. Index de Enfermería [Revista en internet]. Fundación Index; septiembre de 2014 [citado 2 de septiembre de 2015]; 23(3): 162-6. Disponible en: http://scielo.isciii.es/ scielo.php?script= sci_arttext\&pid=S1132-12962014000200010\&Ing= en\&nrm=iso\&tlng=en.

16. Lopez L, Velásquez V, Cataño N, Helena PA, Idaly MA. Por un futuro mejor: programa de promoción de la salud para afrodescendientes. Rev Cuid. 2016; 7(1): 1185-94.

17. Fleckman JM, Dal Corso M, Ramirez S, Begalieva M, Johnson CC. Intercultural Competency in Public Health: A Call for Action to Incorporate Training into Public Health Education. Front public Heal [Revista en internet]. 2015; 3(September): 210. Disponible en: http://www.pubmedcentral.nih. gov/articlerender. fcgi? artid=4556984\&tool=pmcentrez\&rendertype=abstr act.

18. Association of American Medical Colleges. Cultural Competence Education Cultural Competence Education for Medical Students [Revista en internet]. AAMC, editor. Washington; 2005: 17. Disponible en: https://www.aamc.org/ download/54338/ data/culturalcomped.pdf.

19. Kutob RM, Bormanis J, Crago M, Harris JM, Senf J, Shisslak CM. Cultural competence education for practicing physicians: Lessons in cultural humility, nonjudgmental behaviors, and health beliefs elicitation. J Contin Educ Health Prof. 2013; 33(3): 164-73.

20. Gallagher RW, Polanin JR. A meta-analysis of educational interventions designed to enhance cultural competence in professional nurses and nursing students. Nurse Educ Today [Revista en internet]. Elsevier Ltd; 2015; 35(2): 333-40. Disponible en: http://www.sciencedirect.com/science/article/ pii/S0260691714003451.

21. Repo H, Vahlberg T, Salminen L, Papadopoulos I, LeinoKilpi H. The Cultural Competence of Graduating Nursing Students. J Transcult Nurs Off J Transcult Nurs. 2016 feb. Disponible en: http://tcn.sagepub.com/content/ early/2016/02/11/1043659616632046.long.

22. Papadopoulos I, Shea S, Taylor G, Pezzella A, Foley L. Developing tools to promote culturally competent compassion, courage, and intercultural communication in healthcare. J Compassionate Heal Care [Revista en internet]. 2016; 3(1): 2. Disponible en: http://jcompassionatehc. biomedcentral.com/ articles/10.1186/s40639-016-0019-6.

23. Taylor G, Papadopoulos I, Dudau V, Maerten M, Peltegova A, Ziegler M. Intercultural education of nurses and health professionals in Europe (IENE). Int Nurs Rev [Revista en internet]. junio de 2011; 58(2): 188-95. Disponible en: http://doi.wiley.com/10.1111/j.14667657.2011.00892.x.

24. Taylor G, Papadopoulos I, Dudau V, Georges Y, Martin V, Messelis $M$, et al. Intercultural education of nurses and health professionals in Europe 2 (IENE2): Training the trainers. Divers Equal Heal Care. 2013; 10(2): 83-93.

25. Papadopoulos I, Taylor G, Ali S, Aagard M, Akman O, Alpers L-M, et al. Exploring Nurses Meaning and Experiences of Compassion: An International Online Survey Involving 15 Countries. J Transcult Nurs [Revista en internet]. 2015; Disponible en: http:// tcn.sagepub.com/cgi/doi/10.1177/1043659615624740.

26. Red Cuidado Cultural de la salud. Universidad Nacional de Colombia. 2016 Disponible en: http://www. enfermeria.unal.edu.co/redcuidadocultural/. 